\title{
Encrypted With Fuzzy Compliment-Max-Product Matrix in Watermarking
}

\author{
Sharbani Bhattacharya, Associate Professor(IT) \\ IEC-College of Engineering \& Technology, Greater Noida, India
}

\begin{abstract}
Watermark is used to protect copyright and to authenticate images. In digital media, today's world images are in electronic form available in the internet. For its protection and authentication invisible watermarking in encrypted form are used. In this paper encryption is done using fuzzy ComplimentMax-Product matrix and then encrypted watermark is embedded in the digital media at desired places using fuzzy rule. The Region of Interest (ROI) is decided with fuzzification. Then, watermark is inserted at the respective positions in the image. Robustness of watermark is judged for ROI. This method of watermarking is done on all image file formats and it is resistant for geometric, noise and compression attack.
\end{abstract}

Keywords-Watermarking; Fuzzy Compliment-Max-Product Matrix, Fuzzification; Encryption

\section{INTRODUCTION}

Watermark is used to protect copyright and to authenticate images. In digital media, today's world images are in electronic form available in the internet. For its protection and authentication invisible watermark in encrypted form are used. Cryptography is an art of converting a message into cipher text and send to the destination. The authorized person can decipher the text and retrieve the original message. This technology is used from very beginning of the civilization. As the days go by we have new and recent technology coming up. Prior we had texts which are converted into cipher text by using some notion that A should read as B and B should be read as $\mathrm{C}$ and so on. Doing this we get a cipher text which is not easily readable unless one knows the conversion method. Public Key and Private Key method is used for cryptography. There are many methods like RSA, DES, Diffie-Hellmann and etc. for cryptography.

In 1976, Martin Hellman, a professor at Stanford University, and Whitfield Diffie, a graduate student, introduced the concept of asymmetric or public key cryptography. In this paper encryption is done using proposed Fuzzy Compliment-Max-Product matrix composition and then encrypted watermark is embedded in the digital media at desired places using fuzzy rule. The Region of Interest (ROI) is decided with fuzzification. Then, watermark is inserted at the respective positions in the image. Robustness of watermark is judged for ROI. Robustness is concerned about tracing or tampering of watermark by attacker. A good watermark should be against filtering process, noise addition, lossy compression, geometry transformation such as rotation, scaling and translation.
The proposed method of watermarking is done on all image file formats and it is resistant for geometric filters, noise and compression attack.

\section{FuZZY RULES AND COMPLIMENT- MAX-PRODUCT MATRIX}

The Fuzzy rules are consisting of rules defined on fuzzy set. Fuzzy set are acquired from Crisp Set using membership function. This process is known as fuzzification. Converting fuzzy set to Crisp set is called defuzzification. In Fuzzy sets the elements are from 0 to 1 . Here, we will be using fuzzy Compliment-Max-Product matrix for encryption of the text/file. The encrypted file is then embedded into digital image as watermark. The embedding process also involves fuzzy rules. The encrypted watermark can be extracted from the digital image in unified format. The unified format is then decrypted using algorithm. If the watermarked image is tried to tamper or change, the information can be obtained from the image. The proposed rule is Fuzzy Compliment-Max-Product matrix composition. This rule is consisting of following method. Let A, B and C are fuzzy set with $\mathrm{A}(\mathrm{x} 1, \mathrm{x} 2), \mathrm{B}(\mathrm{y} 1, \mathrm{y} 2)$ and $\mathrm{C}(\mathrm{z} 1, \mathrm{z} 2)$.Let us say,

$$
\begin{aligned}
& \mu_{\mathrm{A}, \mathrm{B}}(\mathrm{x} 1, \mathrm{y} 1)=0.2 \\
& \mu_{\mathrm{A}, \mathrm{B}}(\mathrm{x} 1, \mathrm{y} 2)=0.3 \\
& \mu_{\mathrm{A}, \mathrm{B}}(\mathrm{x} 2, \mathrm{y} 1)=0.2 \\
& \mu_{\mathrm{A}, \mathrm{B}}(\mathrm{x} 2, \mathrm{y} 2)=0.4 \\
& \mu_{\mathrm{B}, \mathrm{C}}(\mathrm{y} 1, \mathrm{z} 1)=0.3 \\
& \mu_{\mathrm{B}, \mathrm{C}}(\mathrm{y} 1, \mathrm{z} 2)=0.5 \\
& \mu_{\mathrm{B}, \mathrm{C}}(\mathrm{y} 2, \mathrm{z} 1)=0.2 \\
& \mu_{\mathrm{B}, \mathrm{C}}(\mathrm{y} 2, \mathrm{z} 2)=0.2
\end{aligned}
$$

The matrix of $\mu_{\mathrm{A}, \mathrm{C}}$ is

$\mu_{\mathrm{A}, \mathrm{C}}(\mathrm{x} 1, \mathrm{z} 1)=1-\max \left\{\left[\mu_{\mathrm{A}, \mathrm{B}}(\mathrm{x} 1, \mathrm{y} 1) * \mu_{\mathrm{B}, \mathrm{C}}(\mathrm{y} 1, \mathrm{z} 1)\right],\left[\mu_{\mathrm{A}, \mathrm{B}}\right.\right.$ $\left.\left.(\mathrm{x} 1, \mathrm{y} 2) * \mu_{\mathrm{B}, \mathrm{C}}(\mathrm{y} 2, \mathrm{z} 1)\right]\right\}=0.94$

$\mu_{\mathrm{A}, \mathrm{C}}(\mathrm{x} 1, \mathrm{z} 2)=1-\max \left\{\left[\mu_{\mathrm{A}, \mathrm{B}}(\mathrm{x} 1, \mathrm{y} 1) * \mu_{\mathrm{B}, \mathrm{C}}(\mathrm{y} 1, \mathrm{z} 2)\right],\left[\mu_{\mathrm{A}, \mathrm{B}}\right.\right.$ $\left.\left.(\mathrm{x} 1, \mathrm{y} 2) * \mu_{\mathrm{B}, \mathrm{C}}(\mathrm{y} 2, \mathrm{z} 2)\right]\right\}=0.90$ 
$\mu_{\mathrm{A}, \mathrm{C}}(\mathrm{x} 2, \mathrm{z} 1)=1-\max \left\{\left[\mu_{\mathrm{A}, \mathrm{B}}(\mathrm{x} 2, \mathrm{y} 1) * \mu_{\mathrm{B}, \mathrm{C}}(\mathrm{y} 1, \mathrm{z} 1)\right],\left[\mu_{\mathrm{A}, \mathrm{B}}\right.\right.$ $\left.\left.(\mathrm{x} 2, \mathrm{y} 2) * \mu_{\mathrm{B}, \mathrm{C}}(\mathrm{y} 2, \mathrm{z} 1)\right]\right\}=0.94$

$\mu_{\mathrm{A}, \mathrm{C}}(\mathrm{x} 2, \mathrm{z} 2)=1-\max \left\{\left[\mu_{\mathrm{A}, \mathrm{B}}(\mathrm{x} 2, \mathrm{y} 1) * \mu_{\mathrm{B}, \mathrm{C}}(\mathrm{y} 1, \mathrm{z} 2)\right],\left[\mu_{\mathrm{A}, \mathrm{B}}\right.\right.$ $\left.\left.(\mathrm{x} 2, \mathrm{y} 2) * \mu_{\mathrm{B}, \mathrm{C}}(\mathrm{y} 2, \mathrm{z} 2)\right]\right\}=0.90$

\section{PROPOSED ENCRYPTION ALGORITHM}

The encryption is done using fuzzy set values. The fuzzy rules are then used to decrypt the context. The encryption algorithm has following steps

Step 1: Choose two Fuzzy matrices appropriate for encryption according to the file size.

Step2: Find the Fuzzy Compliment-Max-Product Matrix Composition.

Step3: Generate random number using Fuzzy Matrix.

Step4: Retrieve the encrypted text/files.

There are various ways of encryption. Here, 2X2 fuzzy matrix is used and Compliment-Max-Product of the fuzzy matrix is obtained.

The text/files is encrypted by one of the matrix. File is divided into four parts and a11, a12, a21 and a22. Each part is encrypted using fuzzy matrix values by generating random number using the fuzzy values. files are then used for watermarking.

The encrypted

\section{Deal dinceypt - Notepad}

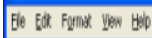

Do not encryot Just insert.

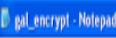

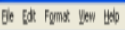

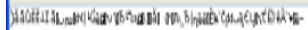

Fig. 2. Encrypted Text to be watermarked

\section{PROPOSED DECRYPTION ALGORITHM}

Decryption algorithm is used decrypt the encrypted file. The following algorithm is used-

Step1: Choose Fuzzy matrix key for decryption coming from encryption algorithm

Step2: Find the Compliment-Max-Product Matrix and break the file into same four parts with appropriate values of fraction.

Step3: Retrieve the original file

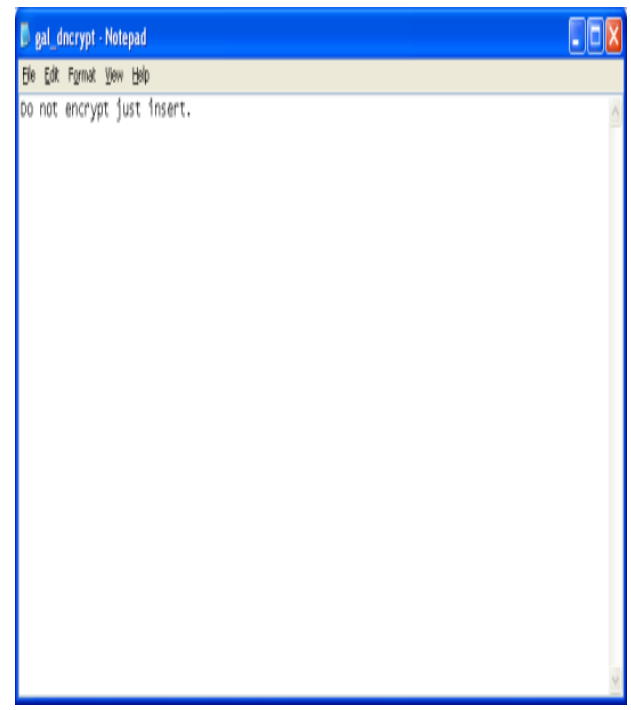

Fig. 3. Decrypted Watermarking File

Fig. 1. Text File containing text to be encrypted 


\section{EMBEDDING THE WATERMARK}

There are various methods of watermarking. Watermark is visible or invisible. Sometimes invisible watermark is cipher text in order to make watermark more robust and not easily identifiable. When encrypted text or file is embedded in the image the watermark is undetectable. The image is divided into parts one region of interest (ROI) and another is nonregion of Interest (i.e. background). Watermarking in the ROI is done where authentication of image is connected.

As we watermark in the ROI then it cannot be easily tampered or deleted. In medical images watermarking is done in non-ROI as to preserve the image accuracy. Here, we will use Fuzzy logic to watermark the image.

The image is having five parts according to ROI
a) High Priority $(H P)$-is the main area
b) Medium Priority (MP)- next important
c) Low Priority(LP)-least important
d) Background( $B G)$ - background of picture
e) Outside (OS)- may be padding zone

All images may not have all five parts. Some may be divided as HP, BG \& OS and so on. It depends on image in how many parts it can be divided. OS is padding i.e. the part of file where picture definition is not stored. This is the nonvisible region of image.The Fuzzification is converting crisp set to fuzzy set with a membership function. Here, we have Crisp Set A consisting the four image zone. The Fuzzy Set A consists of elements with membership function $\mu$. Here, we want to embed watermark at ROI of image. We take $A=\{$ HP,MP,LP, BG $\}$. In case of medical image we take $B=\{$ $\mathrm{BG}, \mathrm{OS}\}$ as crisp set and then fuzzfication is done.

$$
\begin{aligned}
& A=\left\{x_{1}, x_{2}, x_{3}, x_{4}\right\} \\
& \mu_{A}\left(x_{1}\right)=0.44 / \mathrm{HP} \mu_{A}\left(x_{2}\right)=0.42 / \mathrm{MP} \\
& \mu_{A}\left(x_{3}\right)=0.14 / \mathrm{LP} \quad \mu_{A}\left(x_{4}\right)=0.14 / B G
\end{aligned}
$$

The watermark is divided into four fuzzy set elements $\mathrm{x}_{1}, \mathrm{x}_{2}, \mathrm{x}_{3}$ and $\mathrm{x}_{4}$. It is stored in the regions HP,MP ,LP and BG respectively. Fig 4 shows watermarked image with fuzzified encrypted text file. When we want to retrieve the watermark we need to defuzzify and collect the four parts of watermark then its combined to one file. Then, this file is decrypted using decryption algortihm as given above.Different images have different membership functions for embedding like some images have logic like $(0.25 / \mathrm{x} 1,0.25 / \mathrm{x} 2,0.25 / \mathrm{x} 3,0.25 / \mathrm{x} 4)$. The $\mathrm{x} 1$ is starting of embedding, $\mathrm{x} 2, \mathrm{x} 3$ and $\mathrm{x} 4$ are end of ROI of image. In order to make a robust watermarking it should be resistant to noise attack, geometric filter attack and compression attack. Embedding is resistant to all of three above said method.

Steps to be followed for watermarking-
Step1: Deciding the region for embedding using fuzzy membership function.

Step2: Divide encrypted watermark into four files.

Step3 : Convert the Digital Image into Byte Code.

Step4: Convert the Encrypted Watermark files to Byte Code.

Step5: Insert the Byte code of Watermark into Image file using Fuzzy rule.

Step6: Convert the Byte Code to desired Image File Format.

\section{Retrieving The WATERMARK From Digital IMAGE}

Retrieving the watermark is done by extracting watermark from Digital Image and decrypting the file and obtained the watermark. Steps to be followed are as follows-

Step1: Convert the Watermarked file into Byte Code.

Step2: Extract the Byte Code of Watermark using defuzzification.

Step3: Convert the Byte Code file to text/file.

Step4: Decrypt the text/file using Decryption algorithm.

\section{CONCLUSION}

The digital images are watermarked with encrypted files in order to have invisible watermark. The watermark is encrypted and decrypted to see whether image is authentic or it is tried to tamper. The watermark is robust against geometric filter attack, scaling attack, compression attack and noise attack. The drawback of the method is, it uses fraction values for encryption like you encrypt by 0.2 . Now for decryption $0.1,0.2$ and 0.3 values can work out. This is loop hole of fractions as values are nearby. So, appropriate programming is required so that decryption cannot be done with other than expected value or key. The proposed method of watermarking depends upon the type of image on which watermark is used. The medical images, images of natural calamity and weather forecasting, company logo, Software logo and etc have different requirements for authenticity and copyright protection. In medical images and weather forecasting ROI is most important so watermark should be out of ROI. In company logo or Software logo tampering or deletion of watermark is an issue so watermark is to be embedded in ROI.

\section{FUTURE SCOPE OF WORK}

The future scope of work is on invisible watermark, fuzzy rules creation and embedding the watermark so that difference in quality of image of original and watermarked image is minimal. 


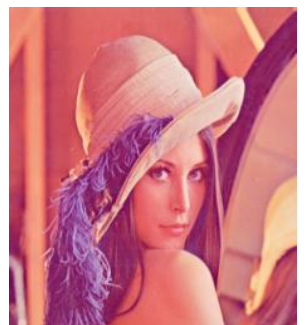

Original Lena.tif

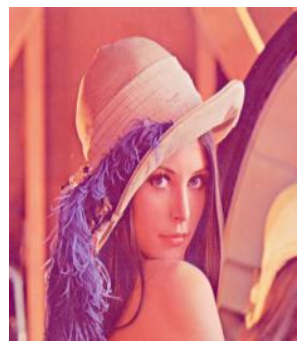

Watermarked Lena.tif

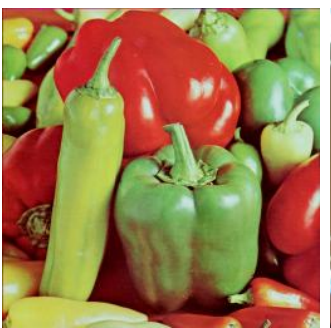

Pepper.tif

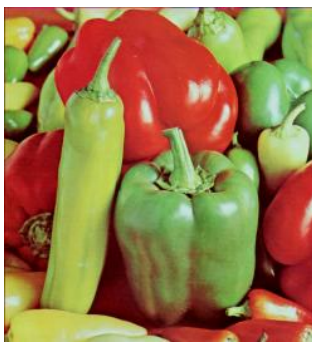

Pepper.tif

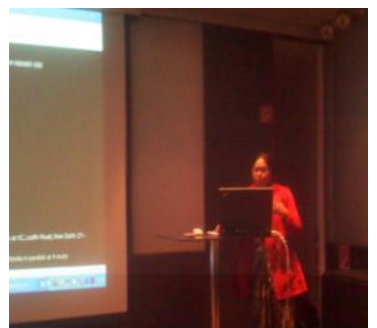

My Pic.jpg

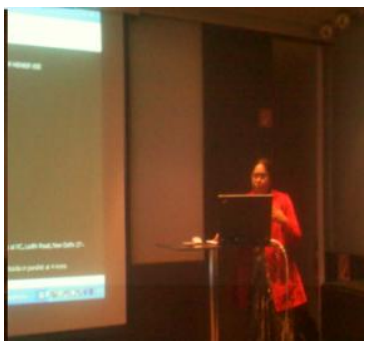

My pic.jpg

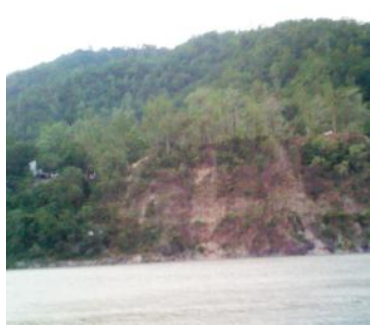

Rishikesh.tif

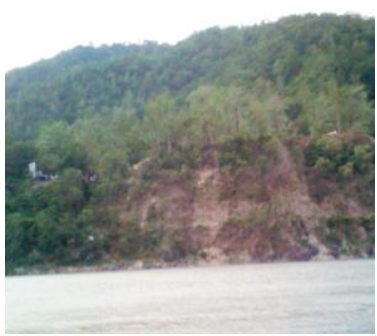

Rishikesk.tif

Fig. 4. Original and Watermarked Images

\section{REFERENCES}

[1] Anandabrata Pal and Nasir Memon, "Evolution of File Carving",Page 59, IEEE Signal Processing Magazine, March 2009.

[2] Anderson Rocha, Walter Scheirer and Terrance Boult, Siome Goldenstein, "Vision of the Unseen: Current Trends and Challenges in Digital Image and Video Forensics" ACM Computing Surveys, Vol. 43, No. 4, Article 26, Publication date: October 2011.

[3] Alain $\operatorname{Tr}$ 'emeau,Shoji Tominaga and Konstantinos N. Plataniotis, Review Article "Color in Image and Video Processing: Most Recent Trends and Future Research Directions", Hindawi Publishing Corporation EURASIP Journal on Image and Video Processing Volume 2008, Article ID 581371, 26 pages ,doi:10.1155/2008/581371.

[4] Alimohammad Latif, " An Adaptive Digital Image Watermarking Scheme using Fuzzy Logic and Tabu Search", Journal of Information Hiding and Multimedia Signal Processing, Volume 4, Number 4, October 2013.

[5] Chuntao WangJiangqun $\mathrm{Ni}$,and Jiwu, "An Informed Watermarking Scheme Using Hidden Markov Model in the Wavelet Domain" Huang,Page853, IEEE Transactions On Information Forensics And Security, Vol. 7, No. 3, June 2012.

[6] Chun-Hsiang Huang, Shang-Chih Chuang, Yen-Lin Huang, and Ja-Ling $\mathrm{Wu}$, "Unseen Visible Watermarking: A Novel Methodology for Auxiliary Information Delivery via Visual Contents", Page193, IEEE Transactions On Information Forensics And Security, Vol. 4, NO. 2, JUNE 2009.

[7] David J. Coumou, Athimoottil Mathew, “A Fuzzy Logic Approach To Digital Image Watermarking”, ,Rochester Institute of Technology.

[8] Dong Zheng, Yan Liu, Jiying Zhao, And Abdulmotaleb El Saddik, "A Survey of RST Invariant Image Watermarking Algorithms", University of Ottawa, ACM Computing Surveys, Vol. 39, No. 2, Article 5, Publication date: June 2007.

[9] E. C. C. Tsang, Changzhong Wang, Degang Chen, Congxin Wu and Qinghua Hu, "Communication Between Information Systems Using Fuzzy Rough Sets", IEEE Transactions On Fuzzy Systems, Vol. 21, No. 3, June 2013, Page 527.
[10] Elzbieta Zielinska, Wojciech Mazurczyk,Krzysztof Szczypiorski, "Trends in Steganography", Communication of ACM, , Vol 57 ,No. 3, March 2014.

[11] Fawad Ahmed, Farook Sattar, Mohammed Yakoob Siyal, and Dan Yu, "A Secure Watermarking Scheme for Buyer-Seller Identification and Copyright Protection" , Hindawi Publishing Corporation EURASIP Journal on Applied Signal Processing Volume 2006, Article ID 56904, Pages 1-15 ,DOI 10.1155/ASP/2006/56904.

[12] Farid "Digital Image Forensic" farid@cs.dartmouth.edu ,www.cs.dartmouth.edu/farid.

[13] Franco Frattolillo,"Watermarking Protocol for Web Context”,Page 350, IEEE Transactions On Information Forensics And Security, Vol. 2, No. 3, September 2007.

[14] G. Fahmy,M. F. Fahmy and U. S.Mohammed, Research Article "Nonblind and Quasiblind Natural Preserve TransformWatermarking" Hindawi Publishing Corporation EURASIP Journal on Advances in Signal Processing,Volume 2010, Article ID 452548, 13 pages doi: $10.1155 / 2010 / 452548$.

[15] Hooman Tahayori, Alireza Sadeghian and Witold Pedrycz, "Induction of Shadowed Sets Based on the Gradual Grade of Fuzziness", ”, IEEE Transactions On Fuzzy System, Vol. 21, No. 5, October 2013,Page 937.

[16] Hua Yuan and Xiao-Ping Zhang,, "Multiscale Fragile Watermarking Based on the Gaussian Mixture Model" Page 3189, IEEE Transactions On Image Processing, Vol. 15, No. 10, October 2006.

[17] Jen-Sheng Tsai, Win-Bin Huang, and Yau-Hwang Kuo, "On the Selection of Optimal Feature Region Set for Robust Digital Image Watermarking", page 735, IEEE Transactions On Image Processing, Vol. 20, No. 3, March 2011.

[18] Mohammad Ali Akhaee,Sayed Mohammad Ebrahim Sahraeian, and Craig Jin, "Blind Image Watermarking Using a Sample Projection Approach" Page 883, IEEE Transactions On Information Forensics And Security, Vol. 6, No. 3, September 2011.

[19] Mriganka Gogoi , H.M.Khalid Raihan Bhuyan ,Koushik Mahanta , Dibya Jyoti Das and Ankita Dutta, "Image and Video based double watermark extraction spread spectrum watermarking in low variance 
region", International Journal of Advanced Computer Science and Applications, Vol. 4, No. 6, July 2013.

[20] Roberto Caldelli, Francesco Filippini, and Rudy Becarelli, Review Article "Reversible Watermarking Techniques:An Overview and a Classification", Hindawi Publishing Corporation EURASIP Journal on Information Security ,Volume 2010, Article ID 134546, 19 pages ,doi:10.1155/2010/134546.

[21] Pankaj U.Lande,S.N. Talbar,G.N. Shinde, "FPGA implementation of image adaptive watermarking using human visual model",ICGSTPDCS, Vol.9,Issue1, Oct. 2009.

[22] Pankaj U.Lande ,Sanjay N. Talbar ,G.N. Shinde, "Robust Image Adaptive Watermarking Using Fuzzy Logic An FPGA Approach", International Journal of Signal Processing, Image Processing and Pattern Recognition, Vol. 3, No. 4, December, 2010.

[23] Peyman Rahmati, Andy Adler and Thomas Tran , "Watermarking in E-commerce", International Journal of Advanced Computer Science and Applications, Vol. 4, No. 6, 2013.

[24] Qian Ying, Ren Xue-mei, Huang Ying and Meng Li, “ Image Sharpness Metric Based on Algebraic Multi- Grid Method",International Journal of Advanced Computer Science and Applications, Vol. 5, No. 4,April 2014.

[25] Sameh Oueslati and Adnane Cherif and Bassel Solaiman, "Maximizing Strength of Digital Watermarks Using Fuzzy Logic”, Signal \& Image
Processing : An International Journal(SIPIJ) Vol.1, No.2, December 2010.

[26] Sameh Oueslati, Adnane Cherif \& Basel Solaiman, "A Fuzzy Watermarking Approach Based on the Human Visual System”, page 218, International Journal Of Image Processing (IJIP), Volume (4): Issue (3) 2010 .

[27] Sharbani Bhattacharya, "Watermarking Digital Image Using Fuzzy Matrix Rules", presented in National Conference Smarter Approaches in Computing, Technology \& Applications SACTA 2014 at ITS ,Mohan Nagar, Ghaziabad, published in Conference Proceedings Page No 343, $19^{\text {th }}$ April 2014.

[28] S. P. Mohanty, O. B. Adamo, and E. Kougianos, "VLSI Architecture of an Invisible Watermarking Unit for a Biometric-Based Security System in a Digital Camera", in Proceedings of the 25th IEEE International Conference on Consumer Electronics (ICCE), 2007, pp. 485-486.

[29] T.Sridevi and S Sameena Fatima,"Digital Image Watermarking using Fuzzy Logic approach based on DWT and SVD”, International Journal of Computer Applications, Vol 74- No.13, July 2013.

[30] Teresa Garcia-Valverde, Alberto Garcia-Sola, Hani Hagras, James A. Dooley,Victor Callaghan and Juan A. Botia, "A Fuzzy Logic-Based System for Indoor Localization Using WiFi in Ambient Intelligent Environments", IEEE Transactions On Fuzzy System, Vol. 21, No. 4, August 2013, Page 702. 